\title{
A Case of Paradoxical Embolic ST-Segment Elevation Myocardial Infarction Triggered by Sleep Apnea
}

\author{
Shingo Kujime, Hidehiko Hara, Yoshinari Enomoto, Hisao Yoshikawa, Hideki Itaya, \\ Mahito Noro, Makoto Suzuki, Masato Nakamura and Kaoru Sugi
}

\begin{abstract}
This report describes an obese 39-year-old man who experienced ST-segment elevation myocardial infarction with total thrombotic occlusion of the right coronary artery. Culprit vessel flow was improved by aspiration. Data suggested that myocardial infarction had resulted from paradoxical embolus via a patent foramen ovale triggered by the Mueller maneuver, which had induced negative intrathoracic pressure following an acute increase of right-heart volume in the context of obesity and sleep-disordered breathing (SDB). Obesity is increasing among younger populations and it represents a risk for SDB and thrombosis. Thus, this mechanism should be included within the differential diagnosis for myocardial infarction in young patients.
\end{abstract}

Key words: paradoxical emboli, patent foramen ovale, sleep-disordered breathing, ST-segment elevation myocardial infarction

(Intern Med 51: 1851-1855, 2012)

(DOI: 10.2169/internalmedicine.51.7069)

\section{Introduction}

ST-segment elevation myocardial infarction (STEMI) typically results from platelet thrombi formed by atherosclerotic changes in coronary arteries (1). More rarely, some cases of STEMI result from non-atherosclerotic causes, including cardiogenic embolism, paradoxical embolism, coronary spasm, or coronary artery dissection (2). The present report describes a case of a patient with STEMI resulting from paradoxical embolus via a patent foramen ovale (PFO) that may have been ultimately triggered by sleep-disordered breathing (SDB).

\section{Case Report}

This case involved a 39-year-old obese man without prior history of medical treatment, familial disease or smoking. While sleeping on a chair bending forward over a desk for approximately 3 hours, he developed persistent left anterior chest pain and presented to our medical center. His electrocardiogram revealed intraventricular conduction disturbance and ST-segment elevation in leads II, III, and aVF. Echocar- diography revealed hypokinesis of the inferior left ventricular wall. STEMI was suspected, leading to urgent hospitalization.

At the initial examination, his consciousness was clear, his height was $173 \mathrm{~cm}$, weight was $113 \mathrm{~kg}$, body mass index (BMI) was $37.5 \mathrm{~kg} / \mathrm{m}^{2}$, blood pressure was $140 / 80$ $\mathrm{mmHg}$, and pulse was 48 beats per minute (regular rhythm). There was no jugular venous distension, abnormal sounds on chest auscultation, abnormal abdominal findings or lower extremity edema. The initial laboratory findings, which were primarily obtained early in the morning of the date of admission while sleeping, are shown in Table 1.

Emergency coronary angiography (CAG) revealed total occlusion with translucency in the mid-right coronary artery (RCA) (Fig. 1a). No abnormal finding was noted in the left coronary artery. STEMI was diagnosed with the RCA as the culprit vessel, and emergency percutaneous coronary intervention (PCI) was performed. Due to concerns regarding potential bradycardia during PCI, a temporary pacemaker was inserted prior to PCI. First, a Runthrough-NS ${ }^{\mathrm{TM}}$ guidewire (Terumo Co., Tokyo, Japan) was passed through the lesion, and the site of the lesion was visualized using an intravascular ultrasound (IVUS) catheter (Atlantis ${ }^{\mathrm{TM}}$, Boston-Scientific 
Table 1. Initial Laboratory Findings

\begin{tabular}{llllll}
\hline White blood cell & $\mathbf{1 5 7 0 0}$ & $\mathbf{\mu L}$ & D-dimer & $\mathbf{3 . 6}$ & $\boldsymbol{\mu g} / \mathbf{d L}$ \\
Red bood cell & 549 & $\times 10^{4} / \mu \mathrm{L}$ & Fibrinogen degradation products & 1.6 & $\mu \mathrm{g} / \mathrm{dL}$ \\
Hemoglobin & 16.7 & $\mathrm{~g} / \mathrm{dL}$ & Protein-S antigen & 83 & $\%$ \\
Hematocrit & 48.1 & $\%$ & Protein-C & 122 & $\%$ \\
Platelet & 29 & $\times 10^{4} / \mu \mathrm{L}$ & Protein-C activator & 110 & $\%$ \\
Total protein & 7.9 & $\mathrm{~g} / \mathrm{dL}$ & Plasminogen activator & 93 & $\%$ \\
Albumin & 4.5 & $\mathrm{~g} / \mathrm{dL}$ & $\beta$ thromboglobulin & 30 & $\mathrm{ng} / \mathrm{mL}$ \\
Aspartate aminotransferase & 28 & $\mathrm{IU}$ & Factor 4 & 20 & $\mathrm{ng} / \mathrm{mL}$ \\
Alanine aminotransferase & 25 & $\mathrm{IU}$ & Factor 8 activater & 126 & $\%$ \\
Lactate dehydrogenase & $\mathbf{5 2 4}$ & $\mathbf{I U}$ & Factor 8 inhibitor & $\mathrm{None}$ & $\mathrm{B} . \mathrm{U} / \mathrm{mL}$ \\
Blood urea nitrogen & 19 & $\mathrm{mg} / \mathrm{dL}$ & Factor 9 inhibitor & $\mathrm{None}$ & $\mathrm{B} . \mathrm{U} / \mathrm{mL}$ \\
Creatinine & 0.96 & $\mathrm{mg} / \mathrm{dL}$ & Thrombin-antithrombin 3 complex & 1.0 & $\mathrm{ng} / \mathrm{mL}$ \\
Sodium & 143 & $\mathrm{mM}$ & Plasmin $\alpha 2-$ plasmin inhibitor complex & 0.3 & $\mu \mathrm{g} / \mathrm{mL}$ \\
Potassium & 3.3 & $\mathrm{mM}$ & Factor 2 activator & 117 & $\%$ \\
Chloride & 105 & $\mathrm{mM}$ & Factor 5 activator & 120 & $\%$ \\
Creatine phosphokinase & $\mathbf{1 9 9}$ & $\mathbf{I U} / \mathbf{L}$ & Factor 7 activator & 126 & $\%$ \\
Creatine kinase-MB & $\mathbf{2 3}$ & $\mathbf{I U} / \mathbf{L}$ & Factor 10 activator & 121 & $\%$ \\
C-reactive protein & $\mathbf{0 . 0 6}$ & $\mathbf{m g} / \mathbf{d L}$ & Anticardiolipin antibody & $<8$ & $\mathrm{U} / \mathrm{mL}$ \\
Total cholesterol & 191 & $\mathrm{mg} / \mathrm{dL}$ & Anticardiolipin $\beta 2$ glycoprotein 1 & $<1.2$ & $\mathrm{U} / \mathrm{mL}$ \\
Low density lipoprotein & 118 & $\mathrm{mg} / \mathrm{dL}$ & Lupus anticoaglant & $\mathrm{none}$ & \\
Triglyceride & 117 & $\mathrm{mg} / \mathrm{dL}$ & & & \\
Fasting glucose & 126 & $\mathrm{mg} / \mathrm{dL}$ & & & \\
Hemoglobin A1c & 5.8 & $\%$ & & & \\
Troponin T & 0.0 & $\mathrm{ng} / \mathrm{mL}$ & & & \\
\hline & & & & &
\end{tabular}

Abnormal values are indicated in bold.

a

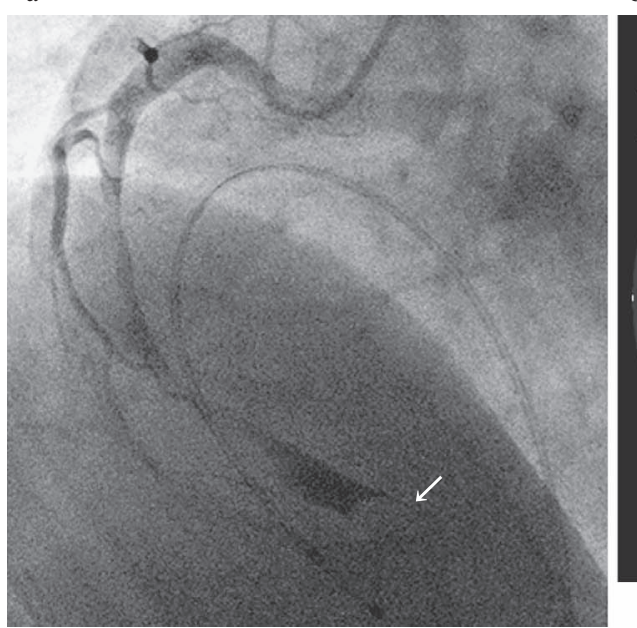

$\mathrm{b}$

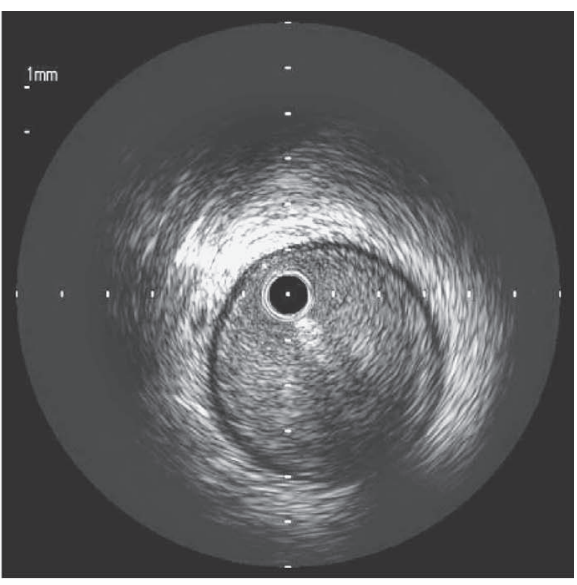

Figure 1. Emergency CAG findings of the RCA, and IVUS findings of the lesion. a: Left panel shows emergency coronary angiographic findings of the RCA. Total occlusion was observed in the distal-RCA (arrow). The site of occlusion was translucent, and the contrast-enhanced image of the RCA distal to the lesion was disrupted. b: Right panel shows IVUS findings of the site of occlusion. The vascular lumen was filled with thrombus. Echoic shadow produced by the guidewire can also be seen. No atherosclerotic changes, such as calcification or plaque rupture, were observed. CAG: coronary angiography, IVUS: intravascular ultrasound, RCA: right coronary artery

Inc., Natick, MA, USA). No atherosclerotic change or atheromatous plaque rupture was noted at the site of the lesion (Fig. 1b). CAG and IVUS findings suggested STEMI caused by thromboembolism to the coronary artery. The Thrombuster-III $^{\circledR}$ aspiration catheter (Kaneka Co., Osaka, Japan) was used for thrombus aspiration, resulting in Thrombolysis in Myocardial Infarction (TIMI) 3 coronary flow and resolution of symptoms. Due to the favorable angiographic result, no other devices, such as balloon, stent or distal protection, were used. Although thrombotic particles were dispersed to peripheral coronary arterioles distal to the lesion (Fig. 2), it was impossible to aspirate all thrombotic particles due to the small diameter of the vessels. We judged that the particles would cause no clinically significant problem due to their small vascular territories, and the PCI procedure was therefore completed. Anticoagulation therapy with warfarin was initiated thereafter. Creatine kinase rose to $1,422 \mathrm{IU} / \mathrm{L}$ during the first 12 hours after PCI and spon- 
a

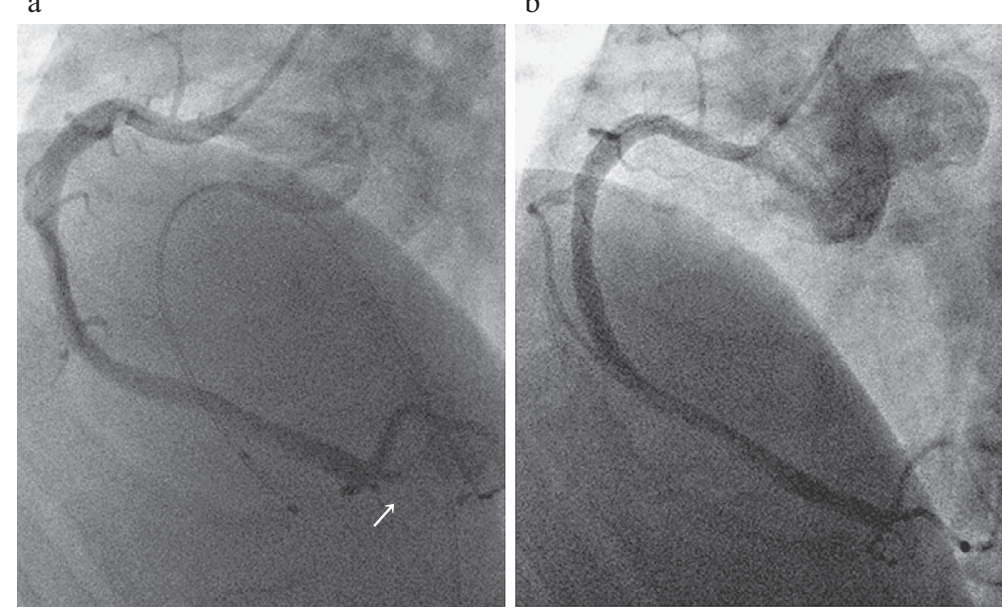

Figure 2. CAG findings of the RCA after thrombus aspiration. a: Left panel shows CAG findings of the RCA after PCI with thrombus aspiration alone. The RCA occlusion resolved with restoration of TIMI 3 coronary flow. However, occlusion due to dispersion of thrombus particles can be seen in coronary arteries distal to the lesion (arrow). b: Right panel shows CAG findings of the RCA obtained 3 months after thrombus aspiration and after 3 months of anticoagulation therapy. The occlusion of peripheral coronary arteries due to dispersion of thrombus particles had resolved.

taneously decreased thereafter. Only mild hypokinesis of the inferior left ventricular wall remained on echocardiography, but this deficit was not thought to interfere with daily activities.

The source of the thrombus was thoroughly investigated. The d-dimer was elevated $(3.6 \mu \mathrm{g} / \mathrm{dL})$ at admission, but no abnormality was observed regarding the levels of coagulation or fibrinolysis or in biomarkers of collagen disease (Table 1). Deep venous thrombosis was not detected by lowerextremity venous ultrasonography at 2 days after the onset of MI. We performed contrast-enhanced computed tomography, but thrombus was not detected in the inferior vena cava, iliac veins, pulmonary arteries, or the vasculature of other organ systems. We also performed CAG with acetylcholine provocation, but there was no sign of coronary vasospasm. He had no history of atrial fibrillation, and he did not develop any atrial fibrillation on ECG monitoring during hospitalization or on 24-hour Holter monitoring during follow-up. Neither intra-cardiac thrombus nor reduced blood flow velocity in the left atrial appendage was detected on transthoracic echocardiography performed on the day of admission or on transesophageal echocardiography (TEE) performed on hospital day 2. Regarding the bubble test during TEE, we found that right-to-left atrial shunt appeared only releasing the Valsalva maneuver, which indicated the presence of PFO (Fig. 3). Because this patient was markedly obese and he was found to have frequent episodes of snoring and apnea during hospitalization, portable cardiopulmonary monitoring was performed using Morpheus ${ }^{\circledR}$ (Teijin Inc., Tokyo, Japan), which yielded an apnea hypopnea index (AHI) of 44.2 (Fig. 4) which suggested underlying SDB.

Warfarin therapy was initiated after PCI, the patient was instructed to lose weight, and continuous positive airway pressure therapy was introduced. He was subsequently dis- charged from the hospital.

\section{Discussion}

This patient had no risk factors for STEMI except for obesity. There was no sign of atherosclerotic change or platelet thrombus formation following plaque rupture at the site of culprit lesion on CAG and IVUS. The cause of STEMI was presumed to be related to paradoxical embolism rather than atherosclerosis. One study reported that $6 \%$ of cases of STEMI did not involve atherosclerotic changes and that $24 \%$ of patients older than 35 years of age with myocardial infarction did not exhibit atherosclerotic changes (2). There are several causes of myocardial infarction in younger patients, including coronary artery thrombus, dissection, spasm, and paradoxical embolism. Coronary vasospasm is considered to be the most common non-atherosclerotic cause of myocardial infarction in Japan (3), but there was no evidence of vasospasm during acetylcholine provocation test in the present case. Further, several cases of paradoxical embolic myocardial infarction have been previously reported (4).

PFO is present in about $25 \%$ of healthy adults $(5,6)$, and there are increasing reports of secondary paradoxical embolism in the context of PFO, particularly with regard to emboli to the brain. Ueno et al. reported that a definitive diagnosis of paradoxical embolism was made in $5 \%$ of cases of acute cerebral infarction (7), while Wechsler et al. reported that PFO was present in about $50 \%$ of young patients with cryptogenic cerebral infarction (8).

In general, the diagnostic criteria for paradoxical embolism include the presence of a right-to-left shunt, as well as: 1) findings of embolism in the arterial system confirmed by imaging; 2) findings of thrombosis in the venous system, 

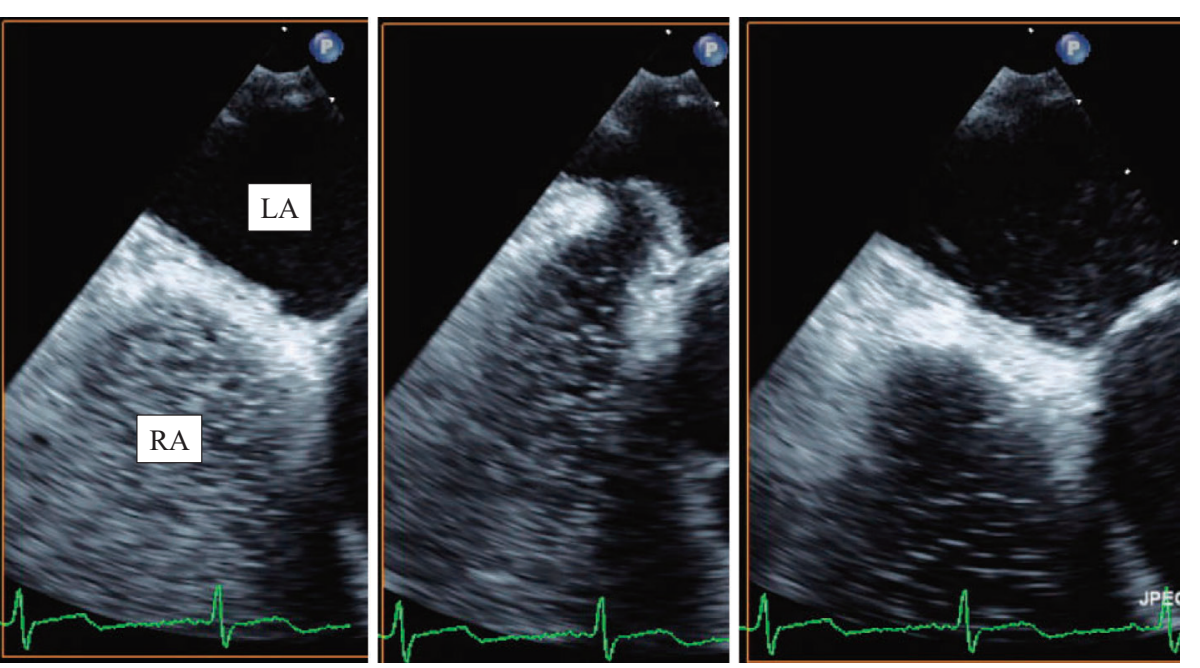

Figure 3. TEE findings. Left panel shows TEE findings prior to a Valsalva maneuver. Bubbles filled the RA, while no particular finding was noted in the LA. The middle panel shows TEE findings during a Valsalva maneuver. Doming of the intra-atrial septum was induced by the maneuver. The right panel shows TEE findings observed at release of the Valsalva maneuver. Bubbles filled the RA and moved through the intra-atrial septum to the LA. LA: left atrium, RA: right atrium, TEE: transesophageal echocardiography

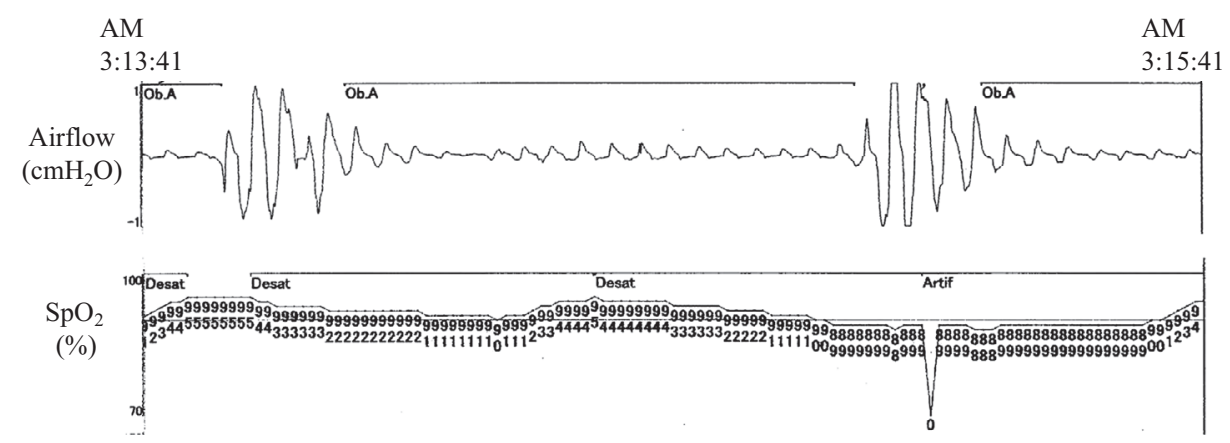

Figure 4. Cardiopulmonary monitoring findings. This figure shows findings of apnea during a 2-minute period of monitoring. As shown in the airflow curve and marker annotation, apnea or hypoventilation that lasted about 1 minute was observed, followed by desaturation on oximetry readings $\left(\mathrm{SpO}_{2}\right)$. Similar findings were frequently observed during the inpatient time. Airflow $\left(\mathrm{cmH}_{2} \mathrm{O}\right)$ shows respiratory curve generated by detection and recording of nasal airflow. $\mathrm{SpO}_{2}(\%)$ shows oxygen saturation. As marker annotations, Ob. A denotes obstructive apnea, and Desat denotes desaturation.

such as deep venous thrombosis or pulmonary thromboembolism; and 3) no source of thrombus formation in the arterial system $(7,9-11)$. Patients with right-to-left shunt who meet all three sub-criteria are defined as "definite" cases, those who meet two of the sub-criteria as "probable" cases, and those who meet one or none of the sub-criteria as "possible" cases (7). Moreover, in our daily clinical practice, the source of thrombus in the right heart system is identified by echocardiography in $\leq 10 \%$ of cases (10) and by magnetic resonance imaging in $20 \%$ of cases (12); thus, there are a number of paradoxical emboli that do not meet the definitive criteria. Since the patient in the present case was obese $\left(\mathrm{BMI}=37.5 \mathrm{~kg} / \mathrm{m}^{2}\right)$ and slept in a chair bending forward for a prolonged period of time, we speculated that the blood in lower extremities stagnated due to physical compression, thereby promoting venous insufficiency and thrombus formation in the venous system (13). This patient had elevated levels of d-dimer at admission, but thrombus was not detected in the venous system, resulting in only a "probable" diagnosis of paradoxical embolism.

Chair-rest position and SDB-associated apnea, both of which were seen in the present case, can cause overload of right heart system and modulation of the intrathoracic pressure via Mueller effects (14). Obesity in young adulthood is increasing in prevalence (15) and represents a risk factor for SDB $(16,17)$, thrombosis (18), and atherosclerosis. Consid- 
ering that PFO is present in approximately $25 \%$ of healthy adults $(5,6)$ and that there is an increasing number of SDB $(19,20)$, non-atherosclerotic causes should be included in the differential diagnosis of STEMI in young adults.

In conclusion, this was a case of paradoxical STEMI considered to have been induced by SDB. Obesity and SDB are increasing in prevalence, and a PFO may play an important role in this pathogenic mechanism. Thus, this phenomenon should be included within the differential diagnosis of STEMI, particularly in young obese patients.

The authors state that they have no Conflict of Interest (COI).

\section{References}

1. Takano T. Guidelines for the management of patients with STelevation myocardial infarction. Circ J 72 (Suppl 4): 1347-1442, 2008 (in Japanese).

2. DeWood MA, Spores J, Notske R, et al. Prevalence of total coronary occlusion during the early hours of transmural myocardial infarction. N Engl J Med 303: 897-902, 1980.

3. Pristipino C, Beltrame JF, Finocchiaro ML, et al. Major racial differences in coronary constrictor response between Japanese and Caucasians with recent myocardial infarction. Circulation 101: 1102-1108, 2000.

4. Murthy A, Shea M, Karnati PK, El-Hajjar M. Rare case of paradoxical embolism causing myocardial infarction: successfully aborted by aspiration alone. J Cardiol 54: 503-506, 2009.

5. Homma S, Sacco RL. Patent Foramen ovale and stroke. Circulation 112: 1063-1072, 2005.

6. Hara H, Virmani R, Ladich E, et al. Patent foramen ovale: current pathology, pathophysiology, and clinical status. J Am Coll Cardiol 46: 1768-1776, 2005.

7. Ueno $\mathrm{Y}$, Iguchi $\mathrm{Y}$, Inoue $\mathrm{T}$, et al. Paradoxical brain embolism may not be uncommon. Prospective study in acute ischemic stroke. J Neurol 254: 763-766, 2007.

8. Wechsler LR. PFO and stroke. What are the data? Cardiol Rev 16:
53-57, 2008.

9. Johnson BI. Paradoxical embolism. J Clin Pathol 4: 316-322, 1951.

10. Lethen H, Flachskampf F, Schneider R, et al. Frequency of deep vein thrombosis in patients with patent foramen ovale and ischemic stroke or transient ischemic attack. Am J Cardiol 80: 1066-1069, 1997.

11. Yasaka M, Otsubo R, Oe H, Minematsu K. Is stroke a paradoxical embolism in patients with patent foramen ovale? Intern Med 44: 434-438, 2005.

12. Cramer S, Rordorf G, Maki J, et al. Increased pelvic vein thrombi in cryptogenic stroke: results of the Paradoxical Emboli from Large Veins in Ischemic Stroke (PELVIS) study. Stroke 35: 46-50, 2004.

13. Greenleaf JE, Rehrer NJ, Mohler SR, Quach DT, Evans DG. Airline chair-rest deconditioning: induction of immobilisation thromboemboli? Sports Med 34: 705-725, 2004.

14. Koshino Y, Villarraga HR, Orban M, et al. Changes in left and right ventricular mechanics during the Mueller maneuver in healthy adults: a possible mechanism for abnormal cardiac function in patients with obstructive sleep apnea. Circ Cardiovasc Imaging 3: 282-289, 2010.

15. Asia Pacific, Cohort Studies. Collaboration. The burden of overweight and obesity in the Asia-Pacific region. Obes Rev 8: 191196, 2007.

16. Olson AL, Zwillich $\mathrm{C}$. The obesity hypoventilation syndrome. Am J Med 118: 948-956, 2005.

17. Mokhlesi B, Tulaimat A. Recent advances in obesity hypoventilation syndrome. Chest 132: 1322-1336, 2007.

18. Abdollahi M, Cushman M, Rosendaal FR. Obesity: risk of venous thrombosis and the interaction with coagulation factor levels and oral contraceptive use. Thromb Haemost 89: 493-498, 2003.

19. Nakayama-Ashida $Y$, Takegami $M$, Chin $K$, et al. Sleep-disordered breathing in the usual lifestyle setting as detected with home monitoring in a population of working men in Japan. Sleep 31: 419-425, 2008.

20. Baldwin CM, Kapur VK, Holberg CJ, Rosen C, Nieto FJ; Sleep Heart Health Study Group. Associations between gender and measures of daytime somnolence in the Sleep Heart Health Study. Sleep 27: 305-311, 2004.

(C) 2012 The Japanese Society of Internal Medicine http://www.naika.or.jp/imindex.html 